\title{
Orbital selective coupling between Ni adatoms and graphene Dirac electrons
}

\author{
M. Gyamfi, ${ }^{1}$ T. Eelbo, ${ }^{1}$ M. Waśniowska,,${ }^{1, *}$ T. O. Wehling $,{ }^{2,},{ }^{*}$ S. Forti, ${ }^{3}$ U. Starke, ${ }^{3}$ \\ A. I. Lichtenstein, ${ }^{2}$ M. I. Katsnelson, ${ }^{4}$ and R. Wiesendanger ${ }^{1}$ \\ ${ }^{1}$ Institute of Applied Physics, University of Hamburg, Jungiusstrasse 11, D-20355 Hamburg, Germany \\ ${ }^{2} 1$ st Institute of Theoretical Physics, University of Hamburg, Jungiusstrasse 9, D-20355 Hamburg, Germany \\ ${ }^{3}$ Max Planck Institute for Solid State Research, Heisenbergstrasse 1, D-70569 Stuttgart, Germany \\ ${ }^{4}$ Institute for Molecules and Materials, Radboud University of Nijmegen, Heijendaalseweg 135, NL-6525 AJ Nijmegen, The Netherlands
}

(Received 13 September 2011; revised manuscript received 20 March 2012; published 11 April 2012)

\begin{abstract}
We investigate the coupling of Ni monomers and trimers to graphene by means of atomically resolved scanning tunneling microscopy (STM). The precise adsorption site of the adatoms is determined experimentally. STM images reveal characteristic nodal structures above the $\mathrm{Ni}$ adatoms and trimers on graphene. First-principles calculations combined with symmetry considerations explain our experimental results by an orbitally controlled interaction of the adatoms and clusters with the Dirac electrons in graphene.
\end{abstract}

DOI: 10.1103/PhysRevB.85.161406

PACS number(s): 73.20.At, 68.37.Ef, 73.20.Hb, 73.22.Pr

The physics and the functionality of nanosystems are largely determined by the coupling of their internal degrees of freedom to the environment. Nanostructures such as adatoms, clusters, or molecules on surfaces can provide charge, ${ }^{1,2}$ spin, ${ }^{3}$ and orbital ${ }^{4-6}$ degrees of freedom and have been investigated on various surfaces. Understanding and controlling their coupling to the surface electrons presents a central goal in nanoscience. Graphene is a two-dimensional material with low-energy electronic excitations resembling massless Dirac fermions, ${ }^{7}$ which makes this material very different from conventional metal surfaces and potentially leads to distinct behavior of adsorbed species.

Numerous theoretical studies have addressed the electronic and magnetic properties of impurities in "Dirac materials" (see, e.g., Refs. 7 and 8) and the problem of transition-metal adatoms on graphene by means of first-principles calculations (cf. Ref. 9 and references therein). A recent scanning tunneling microscopy (STM) experiment has revealed an ionization process of Co adatoms on graphene. ${ }^{2}$ Theoretically, it has been predicted that the orbital degree of freedom can control the coupling of adsorbed species to the graphene electrons, ${ }^{9-13}$ but, to the best of our knowledge, any experimental access to orbital physics of graphene-based nanostructures has not been achieved so far.

In this Rapid Communication, $\mathrm{Ni}$ adatoms and clusters on graphene on $\mathrm{SiC}(0001)$ are investigated by combining STM experiments and theoretical modeling. Our STM measurements demonstrate that $\mathrm{Ni}$ adatoms reside above the hollow site of the graphene lattice. In STM topographies and $\mathrm{d} I / \mathrm{d} U$ maps, we find a peculiar spatial dependence of the local density of the states (LDOS) in the vicinity of $\mathrm{Ni}$ adatoms which is explained by an unconventional orbital-selective coupling between the Ni $3 d$ orbitals and the graphene Dirac fermions. Further STM investigations of Ni trimers on graphene reveal characteristic nodal structures in low-energy $\mathrm{d} I / \mathrm{d} U$ maps, which suggests that graphene can be used as a substrate for resolving subatomic and submolecular structures in local tunneling probe experiments.

All STM and scanning tunneling spectroscopy (STS) measurements were performed at $5 \mathrm{~K}$ in an ultrahigh vacuum system. Ni was deposited onto the sample kept at $12 \mathrm{~K}$. Mono- layer graphene (MLg) was grown on the silicon-terminated $\mathrm{SiC}(0001)$ surface with a doping in the $10^{13} \mathrm{~cm}^{-2}$ regime. ${ }^{14}$ The band structure of MLg on $\mathrm{SiC}(0001)$ was controlled by angle-resolved ultraviolet photoemission spectroscopy before the STM and STS measurements. ${ }^{15}$ First-principles calculations have been performed using the Vienna $a b$ initio simulation package (VASP) ${ }^{16}$ with the implemented projector augmented-wave basis sets ${ }^{17,18}$ and employing a generalized gradient approximation (GGA) ${ }^{19}$ to the exchange correlation potential. We simulated $\mathrm{Ni}$ adatoms on graphene using $3 \times 3$ and $6 \times 6$ graphene supercells. They were relaxed until the forces fell below $0.02 \mathrm{eV}^{-1}$ for each atom. All vacuum LDOS simulations were performed in GGA.

Figure 1(a) presents a STM topography of an epitaxial MLg on $\mathrm{SiC}(0001)$ after the deposition of $\mathrm{Ni}$ atoms onto the cold surface. The STM topography shows the atomically resolved graphene lattice with a superimposed contrast of a hexagonal superlattice, which is caused by the interface carbon buffer layer on the $\mathrm{SiC}(0001)$ substrate underneath the graphene. ${ }^{20}$ Individual $\mathrm{Ni}$ adatoms appear as protrusions with an apparent height of $0.23 \mathrm{~nm} \pm 0.05 \mathrm{~nm}$, depending on the bias voltage and the corrugation of graphene. Interestingly, the topographic measurements reveal a dip above the center of each $\mathrm{Ni}$ adatom, which is in contrast to the generally structureless protrusions observed in STM images of adatoms on metal surfaces [see, e.g., Au adatoms on $\mathrm{Cu}(111)$ in Ref. 21].

To understand these peculiarities in the images of the $\mathrm{Ni}$ adatoms on graphene it is crucial to identify their precise adsorption geometry. Density functional theory (DFT) calculations suggested that $\mathrm{Ni}$ adatoms on freestanding graphene adsorb above the hollow site ( $h$ site) of the graphene lattice (center of the carbon ring). ${ }^{22}$ However, local Coulomb interaction effects as well as the substrate beneath graphene are known to affect the adsorption geometry of transition-metal adatoms on graphene, ${ }^{9,11,23}$ which makes an experimental identification of the adsorption geometry highly desirable. The atomically resolved STM topographies allowed us to determine the adsorption site of various $\mathrm{Ni}$ adatoms. As shown in Fig. 1(b), we find all $\mathrm{Ni}$ adatoms residing in one adsorption site only, i.e., above the $h$ site. This is in line with the theoretical predictions for $\mathrm{Ni}$ on freestanding 


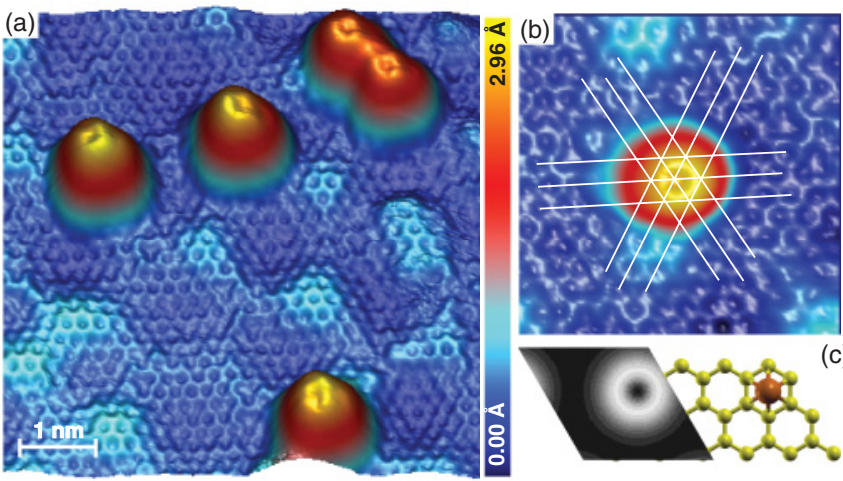

FIG. 1. (Color online) (a) Topographic image of Ni monomers on graphene. Tunneling parameters: $U=200 \mathrm{mV}$ and $I=0.3 \mathrm{nA}$. (b) STM topography indicating the adatom's position on graphene. $U=200 \mathrm{mV}$ and $I=0.3 \mathrm{nA}$. The scan size is $3 \mathrm{~nm} \times 3 \mathrm{~nm}$. (c) Simulated LDOS in the vacuum above a single Ni adatom on graphene (left) and adsorption geometry of the adatom (right). The energy range between the Fermi level and $0.1 \mathrm{eV}$ has been integrated. The LDOS has been calculated at a height of $4.0 \AA$ above the graphene sheet, which corresponds to $2.4 \AA$ above the adatom.

graphene under the assumption of moderate local Coulomb interactions. ${ }^{9,22}$

To unravel the origin of the dip structures observed on the $\mathrm{Ni}$ adatoms [Figs. 1(a) and 1(b)], we performed DFT simulations of $\mathrm{Ni}$ adatoms on graphene and calculated the LDOS in the vacuum above a $\mathrm{Ni}$ adatom on graphene. This LDOS integrated between the Fermi level $\left(E_{\mathrm{F}}=0\right)$ and $+0.1 \mathrm{eV}$ [Fig. 1(c)] shows a dip structure as experimentally observed. Since our DFT calculations reveal no localized Ni impurity resonances within this energy range [see Fig. 2(b)], the current in STM is due to electrons tunneling from graphene to the tip, where the $\mathrm{Ni}$ atomic orbitals modify the graphene states via hybridization. The tunneling current and the vacuum LDOS above the $\mathrm{Ni}$ atoms therefore reflect the hybridization of the $\mathrm{Ni}$ states with the graphene bands. Analyzing the Ni states which contribute to the vacuum LDOS, we find the most significant

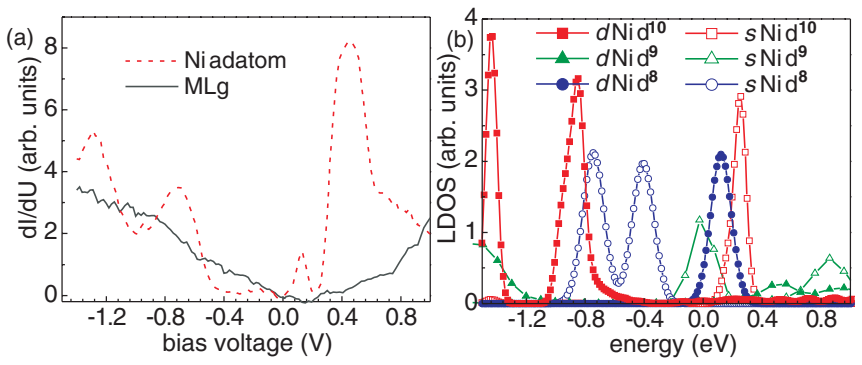

FIG. 2. (Color online) (a) STS spectra measured on the center of the $\mathrm{Ni}$ adatom and bare graphene. Stabilization parameters: $U=1 \mathrm{~V}$ and $I=0.4 \mathrm{nA}$. (b) Simulation of the electronic structure of $\mathrm{Ni}$ monomers in different electronic configurations performed in GGA and GGA $+U$. For the calculations shown here the Ni atoms were placed above the center of a carbon hexagon and an electron doping level of $2 \times 10^{13} \mathrm{~cm}^{-2}$ has been taken into account in the LDOS simulations. contributions from the Ni $3 d_{x z}, 3 d_{y z}, 3 d_{x y}$, and $3 d_{x^{2}-y^{2}}$ orbitals but not from the $4 s$ or $3 d_{z^{2}}$ orbitals, although, in general, the latter two orbitals extend furthest into the vacuum. ${ }^{24}$ Thus, hybridization of the low-energy states and the $\mathrm{Ni} 4 s$ or $3 d_{z^{2}}$ orbitals appears to be suppressed.

In pristine graphene, the low-energy electronic states reside in the corners of the Brillouin zone and-most importantlyare highly symmetric: They transform according to the two-dimensional irreducible representations $E_{1}$ and $E_{2}$ of the crystal symmetry group $C_{6 v} \cdot{ }^{25} \mathrm{~A}$ single $\mathrm{Ni}$ adatom in the center of a carbon hexagon preserves $C_{6 v}$ symmetry. Hence, coupling of adatom orbitals of $E_{1}\left(d_{x z}, d_{y z}\right)$ and $E_{2}$ symmetry $\left(d_{x y}, d_{x^{2}-y^{2}}\right)$ with the graphene Dirac states is most efficient, ${ }^{10,11,13}$ whereas hybridization of the low-energy graphene bands and adatom orbitals such as $\mathrm{Ni} 4 s$ or $\mathrm{Ni} 3 d_{z^{2}}$, which belong to the $A_{1}$ representation of $C_{6 v}$, is suppressed. In contrast to the $\mathrm{Ni} 4 s$ or $\mathrm{Ni} 3 d_{z^{2}}$ orbitals, the orbitals of $E_{1,2}$ symmetry have a node along the $z$ axis, which appears as a dip in the STM images.

Using STS we investigate the local electronic properties of the combined $\mathrm{Ni} /$ graphene system. Within the bias voltage $(U)$ range of -1.3 to $+1.0 \mathrm{~V}$ around the Fermi level we do not find any pronounced peaks on bare graphene, while for the $\mathrm{Ni}$ adatoms we find peaks at $-1.3,-0.7,+0.15$, and $+0.45 \mathrm{~V}$ [see Fig. 2(a)]. The Ni-induced $\mathrm{d} I / \mathrm{d} U$ features reflect the LDOS of the graphene-adatom system and thus carry information about the electronic configuration of the adatoms.

Similar to the case of $\mathrm{Co}, 9,11,12$ also $\mathrm{Ni}$ might appear in different electronic configurations when adsorbed on graphene. ${ }^{9}$ For this reason, we performed DFT calculations of $\mathrm{Ni}$ adatoms on graphene with the adatom predominantly in $d^{8}, d^{9}$, and $d^{10}$ configurations and simulated the LDOS. The different Ni configurations were realized by using GGA (Ni $d^{10}$ configuration) and GGA $+U$ with $U=6 \mathrm{eV}, J=$ $0.9 \mathrm{eV}$ (Ni $d^{8}, d^{9}$ configuration). The $d^{8}$ and $d^{9}$ configurations were obtained by using different starting conditions for the electronic density. Comparing the energy-level spacing of the resonances in experimental and simulated spectra of the $\mathrm{Ni}$ adatoms, we find a good agreement for $\mathrm{Ni}$ in the $d^{10}$ configuration but not for the $d^{8}$ or $d^{9}$ configurations. We further note that only the $d^{10}$ configuration yields the lowest energy for $\mathrm{Ni}$ adatoms above the center of the hexagon as observed experimentally. Thus, there is a strong evidence for Ni being predominantly in the nonmagnetic $d^{10}$ configuration when adsorbed to graphene. We then identify peaks at -0.7 and $-1.3 \mathrm{~V}$ to be $\mathrm{Ni} 3 d$-derived resonances as found in our calculations and Refs. 22 and 26. Furthermore, the pronounced $\mathrm{d} I / \mathrm{d} U$ peak at $+0.45 \mathrm{~V}$ appears to be a $\mathrm{Ni} 4 s$-state derived resonance. In addition, close to the Fermi energy we observed a smaller peak in the $\mathrm{d} I / \mathrm{d} U$ spectra at $+0.15 \mathrm{~V}$. The origin of this peak is unknown and beyond the DFT description of $\mathrm{Ni}$ on freestanding graphene. Possible origins include electronic coupling to the $\mathrm{SiC}(0001)$ buffer layer or many-body effects. Further theoretical calculations are needed to settle this issue.

At energies away from impurity resonances, the spectra and the LDOS of the impurity present a direct fingerprint of the coupling of the impurity to its environment. We therefore measured the energy and spatially resolved LDOS 

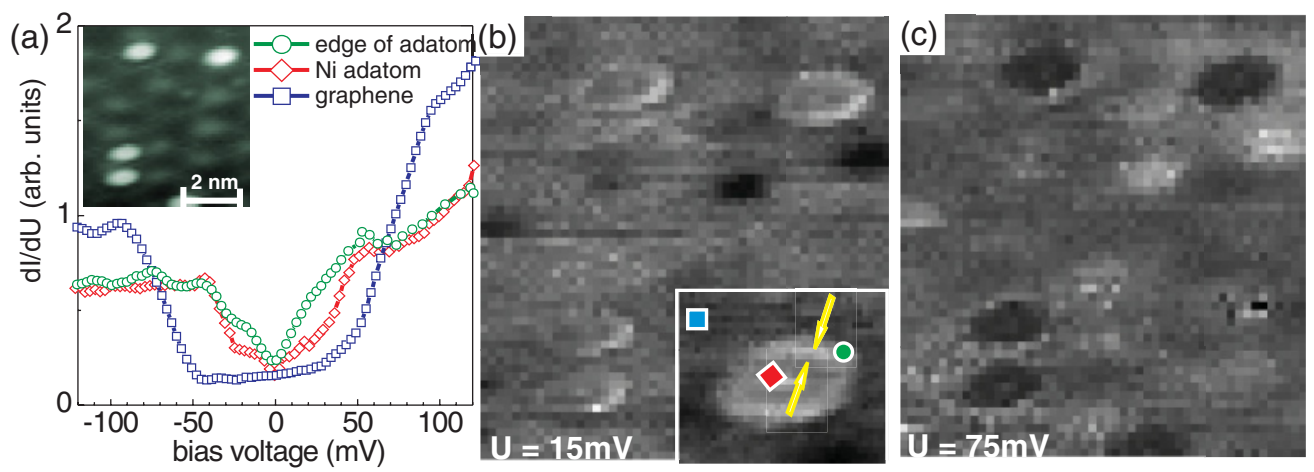

FIG. 3. (Color online) (a) STS spectra of Ni adatom, adatom's edge, and graphene. Tunneling parameters are $U=150 \mathrm{mV}$ and $I=0.3 \mathrm{nA}$. The inset shows a topographic image of graphene coated by Ni adatoms $(U=150 \mathrm{mV}$ and $I=0.3 \mathrm{nA})$. (b) and (c) are spatial maps of the tunneling conductance of the same area presented in the inset of (a). The inset in (b) presents a magnified $\mathrm{d} I / \mathrm{d} U$ map of an adatom and the locations where the spectra were measured. The scan size is $1.7 \mathrm{~nm} \times 1.4 \mathrm{~nm}$.

close to the Fermi energy (Fig. 3), where DFT predicts no $\mathrm{Ni}$ resonances for the $d^{10}$ configuration. For bare graphene two steps around $\pm 70 \mathrm{mV}$ are observed [blue curve, square symbols, in Fig. 3(a)]. This indicates inelastic tunneling assisted by a bosonic mode with $70-\mathrm{meV}$ excitation energy, which can be either $K$-point graphene phonons ${ }^{27-29}$ or resonant coupling to $\mathrm{SiC}(0001)$ buffer layer states. ${ }^{30}$

In the vicinity of the $\mathrm{Ni}$ adatoms the $\pm 70 \mathrm{mV}$ gap disappears and the low-energy LDOS acquires a peculiar position dependence. In the bias range of $-40 \mathrm{mV} \leqslant E \leqslant 40 \mathrm{mV}$, we find a higher $\mathrm{d} I / \mathrm{d} U$ signal at the edge than above the center of the adatom, as experimentally confirmed by a bright ringlike shape at the edge (indicated by yellow arrows) [see Fig. 3(b)]. The enhanced LDOS at the edge of the monomer is well in line with the main contribution to the low-energy LDOS coming from states of $E_{1,2}$ symmetry due to the orbital selective coupling of the $\mathrm{Ni}$ adatoms to the Dirac electrons explained above. We note that the ring structures observed here are different from those reported for Co adatoms on graphene in Ref. 2, which have been attributed to charging of the impurity.

The $\mathrm{d} I / \mathrm{d} U$ spectra measured directly above the Ni adatoms show distinct steps at both positive- and negative-bias voltages, in particular at $E \approx \pm 40 \mathrm{meV}$, which suggest an opening of strong inelastic tunneling channels at this energy. Strong inelastic signals are not surprising in the context of orbital selectively coupled adatom-graphene systems: As the tunneling current for the tip above the adatom is strongly enhanced by $s$-wave admixtures to the adatom LDOS, inelastic tunneling opening any $s$-wave tunneling channel can be expected to lead to pronounced steps in STS-similar to the phonon floodgate observed on pristine graphene. ${ }^{28,31}$ Phonon modes thus present a natural origin of the $\pm 40 \mathrm{meV}$ gap observed above the Ni adatoms, which would also be qualitatively in line with our DFT calculations, revealing vibrational modes of the $\mathrm{Ni}$ adatoms on graphene at $31 \mathrm{meV}^{32}$ Outside the energy window of $\pm 40 \mathrm{meV}$ the opening of an inelastic tunneling channel affects also the real-space appearance of the adatoms in differential conductance maps: The $\mathrm{d} I / \mathrm{d} U$ map at $U=75 \mathrm{mV}$ shows a uniform differential conductance around the $\mathrm{Ni}$ adatoms, which is clearly different from the ring structures at biases below $|U|<40 \mathrm{mV}$. In all cases, we find that the $\mathrm{d} I / \mathrm{d} U$ signal is localized to an area of $1 \mathrm{~nm}$ in diameter, comparable to the apparent lateral extent of the adatoms in the STM topography.

So far, we showed that the behavior of isolated Ni adatoms on graphene is largely controlled by real-space symmetries. To address how less symmetric and more complex structures couple to the graphene electrons, we also investigated $\mathrm{Ni}$ trimers on graphene (Fig. 4) and performed DFT simulations. In our STM topography, the Ni trimer [Fig. 4(a)] appears as a uniform bright protrusion and does not display any dip feature on the centers of individual atoms - in contrast to the constant current image of monomers presented in Fig. 1(a). We attribute this behavior to the reduced symmetry in the case of the trimer: Our DFT calculations yield the relaxed configuration of $\mathrm{Ni}_{3}$ on graphene with the $\mathrm{Ni}$ atoms nearly above the centers of adjacent $C$ hexagons [Fig. 4(d)], thus forming an equilateral triangle. Therefore, the sixfold symmetry of the single adatom on graphene is broken here, while a $C_{3 v}$ threefold rotational symmetry about the center of the trimer emerges [Fig. 4(d)]. As the $\mathrm{Ni}$ atoms of the trimer are off the $C_{3 v}$-rotational symmetry axis, the graphene bands may now couple to any $\mathrm{Ni}$ atomic orbital. This explains the disappearance of the dip structure, which has been attributed to the exclusive coupling between the $E_{1,2}$ adatom orbitals and the graphene bands in the case of monomers.

We further characterized the trimer by means of $\mathrm{d} I / \mathrm{d} U$ maps at different bias voltages. In the bias range $(|U|>$ $30 \mathrm{mV}$ ) the trimer appears as a uniform bright protrusion, as shown for a bias voltage of $-55 \mathrm{mV}$ in Fig. 4(b). This $\mathrm{d} I / \mathrm{d} U$ map is very similar to the topographic image [cf. Figs. 4(a) and 4(b)]. In the $\mathrm{d} I / \mathrm{d} U$ map very close to the Fermi energy, i.e., at $-1 \mathrm{mV}$, the trimer appears, however, as a local minimum with triangular (or " $Y$ ") shape which is surrounded-similar to the monomer-by a "rim" structure with higher LDOS [cf. Figs. 4(c) and 3(b)].

We can understand this behavior by analyzing $\mathrm{Ni}_{3}$ molecular orbitals and their coupling to the graphene states. The center of the trimer is directly above a carbon atom and the low-energy graphene bands decompose into three symmetry classes $\left(E, A_{1}\right.$, and $\left.A_{2}\right)$ under the $C_{3 v}$ symmetry of the trimer. 

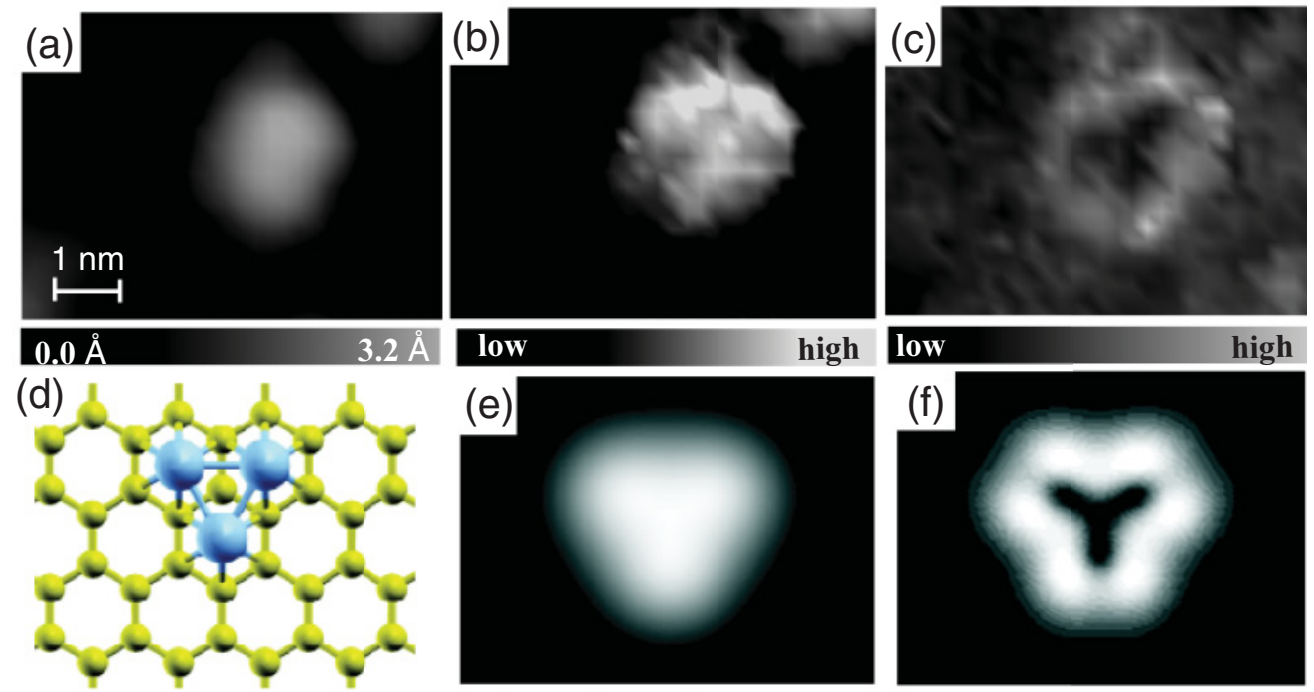

FIG. 4. (Color online) (a) Constant current image of a Ni trimer and corresponding atomic structure in (d) $(U=150 \mathrm{mV}$ and $I=0.3 \mathrm{nA})$. (b) and (c) $\mathrm{d} I / \mathrm{d} U$ maps of the Ni trimer taken at -55 and $-1.0 \mathrm{meV}$, respectively. (e) and (f) calculated LDOS maps on the trimer for $A$ - and $E$-symmetry states.

Thus, $\mathrm{Ni}_{3}$ orbitals of these symmetries can couple to the Dirac fermions in graphene. Our calculations suggest that $\mathrm{Ni}_{3}$ provides electronic states of various symmetries: The simulated low-energy LDOS of the trimer exhibits for instance contributions from states of $E$ and $A$ symmetry [see Figs. 4(e) and 4(f)]. The obtained spatial shape of the twofold degenerate $E$-symmetry states [Fig. 4(f)] agrees very well with the $\mathrm{d} I / \mathrm{d} U$ map measured at $-1 \mathrm{mV}$. At higher energies the calculated LDOS is dominated by $A$-symmetry states. The $\mathrm{d} I / \mathrm{d} U$ map at $-55 \mathrm{mV}$ [in Fig. 4(b)] as well as the integrated LDOS [topography in Fig. 4(a)] do not display ring structures, which is in line with the calculations in Fig. 4(e). Our calculations show that the $A$-symmetry states leak out into the vacuum much stronger than the $E$-symmetry states. Thus, our experimental finding of structurally nontrivial states close to the Fermi level can be understood if a $\mathrm{Ni}_{3}$ resonance of $E$ character close to the Fermi level is assumed.

In conclusion, we report direct imaging of $3 d$ orbitals of transition-metal adatoms on graphene, which is facilitated by the highly symmetric nature of the graphene Dirac electrons. Its peculiar electronic structure makes graphene thus ideally suited for the observation of subatomic structures in local probe measurements.

Financial support from the DFG (Grant No. WI 1277/25), SPP 1459, SFB 668, the Cluster of Excellence "Nanospintronics" (LExI Hamburg), the ERC Advanced Grant FURORE and FOM (The Netherlands), as well as computer time from HLRN are acknowledged. We thank A. Balatsky, A. Rosch, and $\mathrm{T}$. Tudorovskiy for useful discussions.
*Corresponding authors: mwasniow@physnet.uni-hamburg.de; wehling@itp.uni-bremen.de

†Present address: Institut für Theoretische Physik, Universität Bremen, Otto-Hahn-Allee 1, D-28359 Bremen, Germany; Bremen Center for Computational Materials Science, Am Fallturm 1a, D-28359 Bremen, Germany.

${ }^{1}$ N. A. Pradhan, N. Liu, C. Silien, and W. H. Ho, Phys. Rev. Lett. 94, 076801 (2005).

${ }^{2}$ V. W. Brar, R. Decker, H.-M. Solowan, Y. Wang, L. Maserati, K. T. Chan, H. Lee, Ç. O. Girit, A. Zettl, S. G. Louie et al., Nat. Phys. 7, 43 (2011).

${ }^{3}$ F. Meier, L. Zhou, J. Wiebe, and R. Wiesendanger, Science 320, 82 (2008).

${ }^{4}$ H. Brune and P. Gambardella, Surf. Sci. 603, 1812 (2008).

${ }^{5} \mathrm{~K}$. von Bergmann, M. Bode, A. Kubetzka, M. Heide, S. Blügel, and R. Wiesendanger, Phys. Rev. Lett. 92, 046801 (2004).

${ }^{6}$ J. Repp and G. Meyer, Phys. Rev. Lett. 94, 026803 (2005).

${ }^{7}$ A. H. C. Neto, F. Guinea, N. M. R. Peres, K. S. Novosolev, and A. K. Geim, Rev. Mod. Phys. 81, 109 (2009).
${ }^{8}$ A. V. Balatsky, I. Vekhter, and J. X. Zhu, Rev. Mod. Phys. 78, 373 (2006).

${ }^{9}$ T. O. Wehling, A. I. Lichtenstein, and M. I. Katsnelson, Phys. Rev. B 84, 235110 (2011).

${ }^{10}$ T. O. Wehling, H. P. Dahal, A. I. Lichtenstein, M. I. Katsnelson, H. C. Manoharan, and A. V. Balatsky, Phys. Rev. B 81, 085413 (2010).

${ }^{11}$ T. O. Wehling, A. V. Balatsky, M. I. Katsnelson, A. I. Lichtenstein, and A. Rosch, Phys. Rev. B 81, 115427 (2010).

${ }^{12}$ D. Jacob and G. Kotliar, Phys. Rev. B 82, 085423 (2010).

${ }^{13}$ B. Uchoa, L. Yang, S.-W. Tsai, N. M. R. Peres, and A. H. C. Neto, e-print arXiv:1105.4893.

${ }^{14}$ K. V. Emtsev, A. Bostwick, K. Horn, J. Jobst, G. L. Kellogg, L. Ley, J. L. McChesney, T. Ohta, E. Reshanov, E. Rotenberg et al., Nat. Mater. 8, 203 (2009).

${ }^{15}$ C. Riedl, A. A. Zakharov, and U. Starke, Appl. Phys. Lett. 93, 033106 (2008)

${ }^{16}$ G. Kresse and J. Hafner, J. Phys.: Condens. Matter 6, 8245 (1994). 
${ }^{17}$ P. E. Blöchl, Phys. Rev. B 50, 17953 (1994).

${ }^{18} \mathrm{G}$. Kresse and D. Joubert, Phys. Rev. B 59, 1758 (1999).

${ }^{19}$ J. P. Perdew, J. A. Chevary, S. H. Vosko, K. A. Jackson, M. R. Pederson, D. J. Singh, and C. Fiolhais, Phys. Rev. B 46, 6671 (1992).

${ }^{20}$ C. Riedl, U. Starke, J. Bernhardt, M. Franke, and K. Heinz, Phys. Rev. B 76, 245406 (2007).

${ }^{21}$ V. Madhavan, W. Chen, T. Jamneala, M. F. Crommie, and N. S. Wingreen, Science 280, 567 (1998).

${ }^{22}$ C. Cao, M. Wu, J. Jiang, and H. P. Cheng, Phys. Rev. B 81, 205424 (2010).

${ }^{23}$ P. J. Feibelman, Phys. Rev. B 77, 165419 (2008).

${ }^{24}$ The $z$ axis is defined to be perpendicular to the graphene sheet.

${ }^{25}$ D. M. Basko, Phys. Rev. B 78, 125418 (2008).

${ }^{26}$ E. J. G. Santos, A. Ayuela, S. B. Fagan, J. Mendes Filho, D. L. Azevedo, A. G. Souza Filho, and D. Sánchez-Portal, Phys. Rev. B 78, 195420 (2008).
${ }^{27}$ V. W. Brar, S. Wickenburg, M. Panlasigui, C.-H. Park, T. O. Wehling, Y. Zhang, R. Decker, C. Girit, A. V. Balatsky, S. G. Louie et al., Phys. Rev. Lett. 104, 036805 (2010).

${ }^{28}$ Y. Zhang, V. W. Brar, F. Wang, C. Girit, Y. Yayon, M. Panlasigui, A. Zettl, and M. F. Crommie, Nat. Phys. 4, 627 (2008).

${ }^{29}$ Y. Liu, L. Zhang, M. K. Brinkley, G. Bian, T. Miller, and T.-C. Chiang, Phys. Rev. Lett. 105, 136804 (2010).

${ }^{30}$ J. Červenka, K. van de Ruit, and C. F. J. Flipse, Phys. Rev. B 81, 205403 (2010).

${ }^{31}$ T. O. Wehling, I. Grigorenko, A. I. Lichtenstein, and A. V. Balatsky, Phys. Rev. Lett. 101, 216803 (2008).

${ }^{32}$ Vibration spectra were calculated by means of density functional perturbation theory. During the latter calculations, the Ni adatoms were allowed to move freely. During both the relaxations and the phonon calculations the supercell Brillouin zone integration was performed on a $4 \times 4 \times 1$ mesh using $0.1-\mathrm{eV}$ Gaussian broadening for the $6 \times 6$ supercells and $8 \times 8 \times 1$ meshes for the $3 \times 3$ supercells. 